\title{
Highly Charged Ion Trapping and Cooling
}

\author{
L. Gruber, B. R. Beck, J. Steiger, D. Schneider, \\ J. P. Holder, D. A. Church
}

This paper was prepared for submittal to the

15th International Conference on the Application of Accelerators in Research and Industry Denton, TX

November 4-7, 1998

October 22, 1998

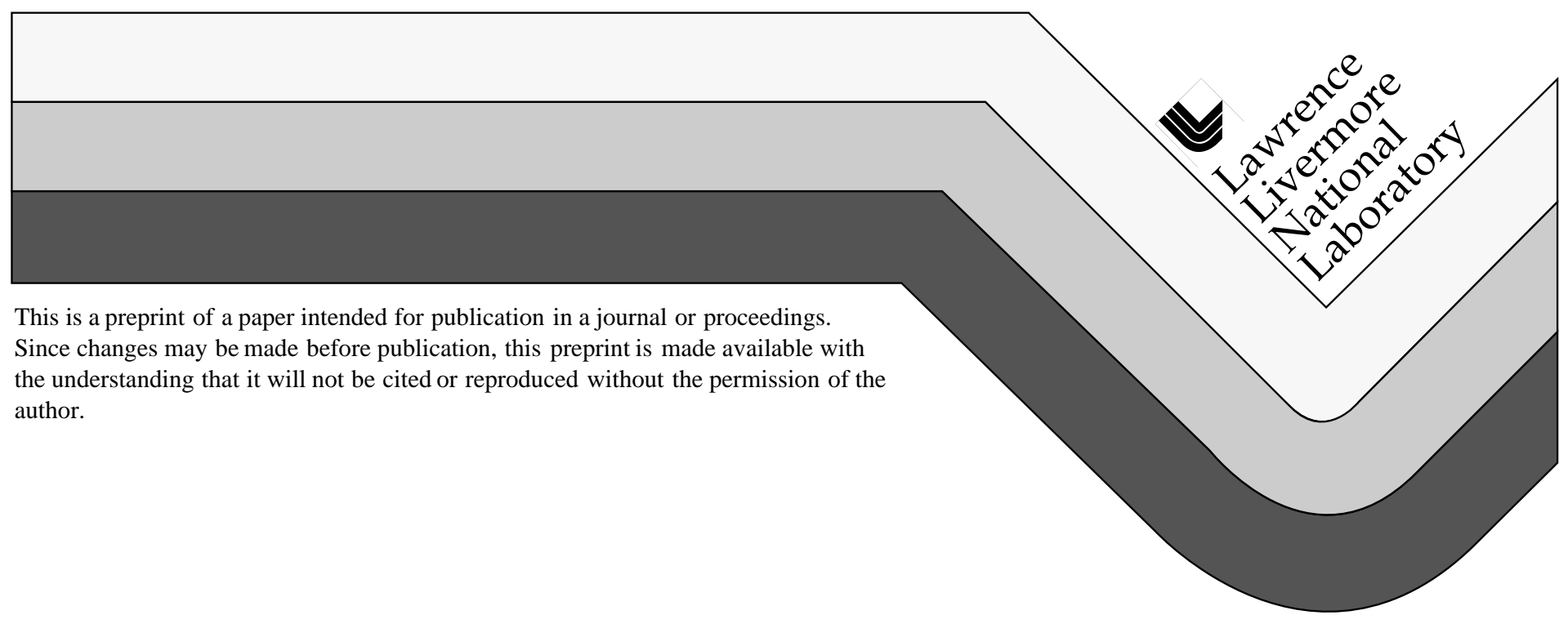




\section{DISCLAIMER}

This document was prepared as an account of work sponsored by an agency of the United States Government. Neither the United States Government nor the University of California nor any of their employees, makes any warranty, express or implied, or assumes any legal liability or responsibility for the accuracy, completeness, or usefulness of any information, apparatus, product, or process disclosed, or represents that its use would not infringe privately owned rights. Reference herein to any specific commercial product, process, or service by trade name, trademark, manufacturer, or otherwise, does not necessarily constitute or imply its endorsement, recommendation, or favoring by the United States Government or the University of California. The views and opinions of authors expressed herein do not necessarily state or reflect those of the United States Government or the University of California, and shall not be used for advertising or product endorsement purposes. 


\title{
Highly Charged Ion Trapping and Cooling
}

\author{
L. Gruber*, B. R. Beck*, J. Steiger*, D. Schneider ${ }^{*}$, J. P. Holder ${ }^{+}$, D. A. Church ${ }^{+}$ \\ *Lawrence Livermore National Laboratory, Livermore, CA 94550 \\ ${ }^{+}$Physics Department, Texas A\&M University, College Station, TX 77843-4242
}

\begin{abstract}
In the past few years a cryogenic Penning trap (RETRAP) has been operational at the Electron Beam Ion Trap (EBIT) facility at Lawrence Livermore National Laboratory. The combination of RETRAP and EBIT provides a unique possibility of producing and re-trapping highly charged ions and cooling them to very low temperatures. Due to the high Coulomb potentials in such an ensemble of cold highly charged ions the Coulomb coupling parameter (the ratio of Coulomb potential to the thermal energy) can easily reach values of 172 and more. To study such systems is not only of interest in astrophysics to simulate White Dwarf star interiors but opens up new possibilities in a variety of areas (e.g. laser spectroscopy, cold highly charged ion beams).
\end{abstract}

\section{INTRODUCTION}

Cold highly charged ion (HCI) plasmas are of interest in spectroscopy, astrophysics and ion beam physics. Fine and hyperfine transitions can potentially be measured to an unprecedented precision. In ion beam physics the reduction of phase space can lead to the development of high brilliance ions beams (1) and in astrophysics these kind of plasmas can simulate the interior of White Dwarf stars (2). The Coulomb coupling parameter $\Gamma$, a parameter classifying how strongly coupled the plasma is, is the ratio of the Coulomb potential energy to the thermal energy of the ions. One-component singly-charged ion crystals have been observed (3). Theory predicts (4) a fluid-solid transition at $\Gamma=172$. In the following, efforts to create strongly coupled plasmas with HCIs are described.

\section{EXPERIMENTAL SETUP}

\section{EBIT and Beam Transport}

An Electron Beam Ion Trap (EBIT) produces ions by successive electron impact ionization (5). These ions are confined axially by static electric fields and radially by the space charge of the electron beam. The ions can be extracted by ramping up the middle drift tube potential (6) (Fig. 1). The extracted ion bunch is guided with static electric and magnetic ion optical elements (Fig. 2) to RETRAP, a cryogenic Penning trap. A $90^{\circ}$ bending magnet in the beamline selects only ions with the same mass to charge ratio for transport to RETRAP. A typical ion bunch is about $5 \mu$ s long and contains $4 \cdot 10^{4} \mathrm{Xe}^{44+}$ ions at an energy of $2 \mathrm{keV} / \mathrm{u}$.

At RETRAP the ions are decelerated upon entering a tube biased at approximately the extraction potential. The tube potential is then rapidly (10 ns) switched to ground thereby reducing the ion kinetic energy to $\approx 70 \mathrm{eV} / \mathrm{u}$. These ions can then be trapped by pulsing a capture electrodes of RETRAP.

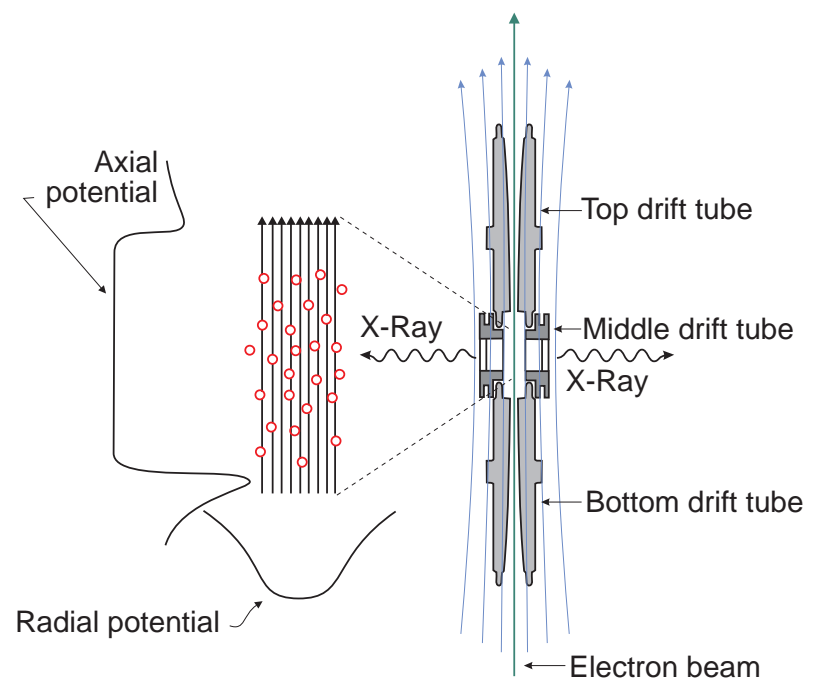

FIGURE 1. Potentials on the three drift tubes create the axial trap in EBIT and the electron beam (indicated by the arrow in the center) creates the radial potential. The potential of the bottom drift tube is higher than the top drift tube potential to assure that the ions escape through the top drift tube when the middle drift tube potential is ramped up. 


\section{RETRAP}

RETRAP (7) is a set of cryogenic Penning traps with a magnetic induction $B$ set to $4.4 \mathrm{~T}$. One of these traps has been used for the experiments described below. This trap has hyperbolic electrodes for electric potential harmonicity and holes in the ring electrode for laser beams and fluorescence light detection. For ion capture and release additional electrodes have been installed above and below the endcap electrodes (Fig. 3).

An electrostatic potential $V_{0}$ applied between the ring and the endcap electrodes produces a potential $V(\rho, z)$ in-

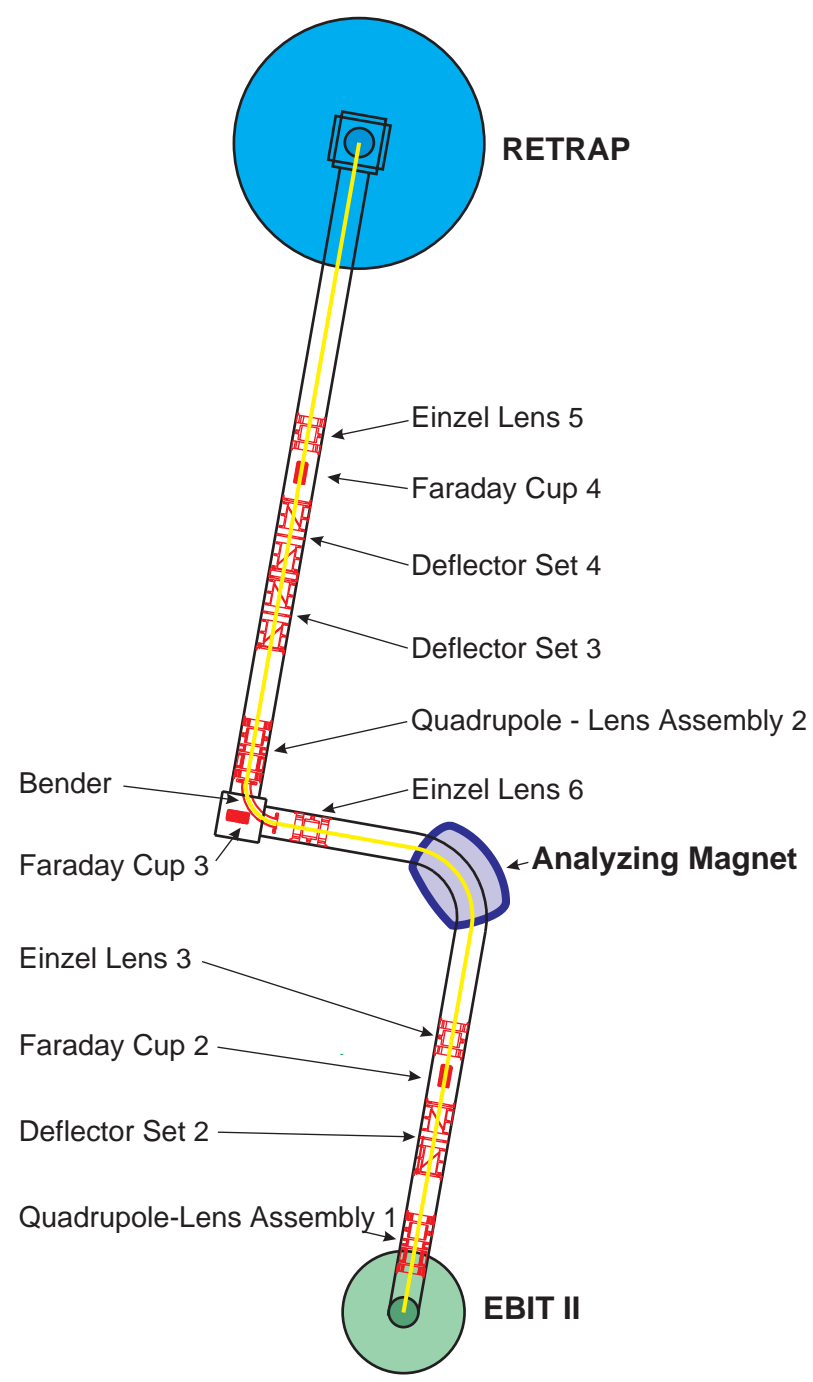

FIGURE 2. Top view of the beamline connecting EBIT II with RETRAP. The path of the ions is out of the paper plane from EBIT II and into the paper plane at RETRAP. side the trap which can be described as

$$
V(\rho, z)=V_{0} \frac{z^{2}-\rho^{2} / 2}{2 d^{2}}
$$

where $z$ is the axial (parallel to the magnetic field) and $\rho$ the radial coordinate. The characteristic length $d$ depends on the half length $z_{0}$ and the radius $\rho_{0}$ of the trap and is given by $d^{2}=\frac{1}{2} \cdot\left(z_{0}^{2}+\frac{\rho_{0}^{2}}{2}\right)$.

The motion of an ion with charge $q$ and mass $m$ in such a potential is a superposition of three independent harmonic oscillations: an axial oscillation along the magnetic field lines with the frequency $\omega_{z}$,

$$
\omega_{z}=\sqrt{\frac{4 q V_{0}}{m d^{2}}}
$$

and two radial oscillations with the frequencies $\omega_{ \pm}$

$$
\omega_{ \pm}=\frac{\omega_{c}}{2} \pm \sqrt{\frac{\omega_{c}^{2}}{4}-\frac{\omega_{z}^{2}}{2}} .
$$

Here $\omega_{c}=\frac{q B}{m}$ is the cyclotron frequency. Equations (2) and (3) are valid only for a single ion moving in the trap. If there are more ions in the trap the potential $V(\rho, z)$ is altered by the space charge of the ions and all frequencies shift by small amounts. Also, when ions are hot and oscillate with large amplitudes, anharmonicities in the trap potential cause broadening and shifts in the resonances.

To detect the axial motion of the ions the trap endcap electrodes are connected with an inductor designed to form a tuned circuit with high quality factor $Q$ and the resonance frequency $\omega_{0}$. The axial motion is detected by tuning the oscillation frequency $\omega_{z}$ of the ions to the resonance frequency $\omega_{0}$ by setting the potential $V_{0}$. An amplifier coupled capacitively to the inductor picks up the

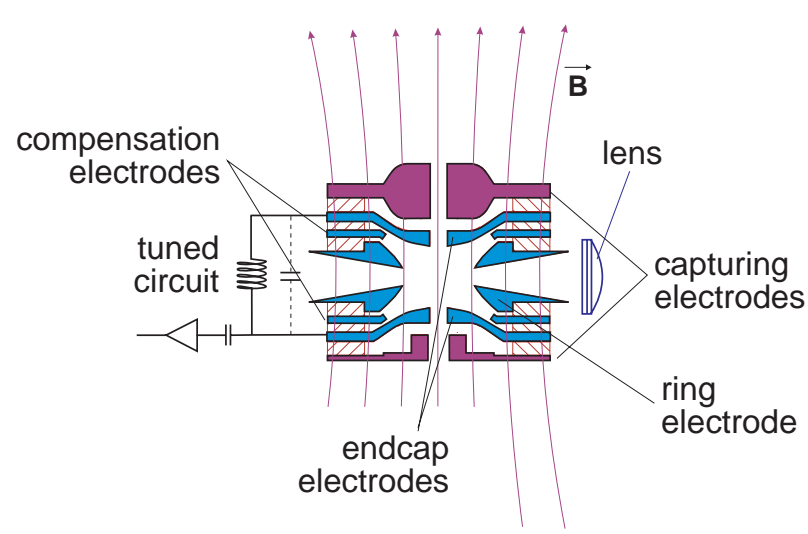

FIGURE 3. Schematic of the hyperbolic trap used in the described experiments. The lens increases the solid angle for fluorescence light detection. 
increased noise signal $V_{S}$ produced by the ions confined in the trap:

$$
V_{S}^{2}=K N Q^{2} q^{2} \frac{U}{m}
$$

where $K$ is a constant for a specific trap geometry, $U$ is the average kinetic energy per ion, $q$ is the charge state, $m$ is the mass and $N$ is the number of the ions on resonance.

This technique probes the trap content nondestructively. Provided the number of ions in the trap does not change, the change of temperature over time can be measured. If the temperature is kept constant the ion number can be determined. Assuming all the ions are in thermal equilibrium the relative numbers of different ion species and charge states in the trap can be determined as well.

\section{COOLING TECHNIQUES}

Singly charged ions with strong electric dipole transitions near the visible have been laser cooled to temperatures below $10 \mathrm{mK}(8)$. Direct laser cooling is not feasible for HCIs since there are no electric dipole transitions accessible with laser light. Different techniques are needed to cool the HCIs to temperatures below $1 \mathrm{~K}$. One possible scheme is to merge the HCIs with a laser cooled $\mathrm{Be}^{+}$ cloud and cool the HCIs by Coulomb collisions (sympathetic cooling) to the temperature of the $\mathrm{Be}^{+}$ions. Different cooling techniques are described below.

An ion oscillating in the trap induces a current in the tuned circuit. If the ion oscillation frequency matches the tuned circuit frequency, the energy of the ion is dissipated in the ohmic resistance of the circuit. The result is an exponential decrease in the ion energy with a time constant $\tau_{R}$ of

$$
\tau_{R}=\frac{4 m z_{0}^{2}}{Q \beta^{2} q^{2} \omega_{0} L}
$$

where $L$ is the inductance of the circuit and $\beta$ is a coupling constant ( $\beta \approx 0.8$ for this trap geometry) (9).

For RETRAP $\left(Q=400, \omega_{0}=2 \pi \cdot 2.5 \mathrm{MHz}, L=\right.$ $200 \mathrm{mH}$ and $z_{0}=5 \cdot 10^{-3} \mathrm{~m}$ ) the calculated cooling time of $\mathrm{Be}^{2+}$ is $\tau_{R}=18 \mathrm{~s}$. It should be noted that only the axial degree of freedom of this particular ion species is cooled in this process and the radial degrees of freedom and other ion species are cooled by collisions among the $\mathrm{Be}^{+}$. This, as well as the fact that not all the ions are on resonance with the tuned circuit, will increase the time for the resistive cooling. Since $\tau_{R}$ is proportional to $m / q^{2}$ the time constant drops rapidly for ions with higher charge states. For $\mathrm{Xe}^{44+}$ this time constant is only $0.56 \mathrm{~s}$.

Laser cooling in RETRAP is done by shining a laser beam into the trap through the holes in the ring, perpendicular to the magnetic field. Therefore the laser cools the two radial degrees of freedom of $\mathrm{Be}^{+}$, when tuned to a wavelength just above that of the resonance transition. If the laser wavelength is tuned below the resonance, heating occurs.

Resistive cooling becomes more effective for higher charges [Eq. (5)], but the cooling limit is given by the temperature of the tuned circuit (4.2 K for RETRAP) (9).

\section{RESULTS}

\section{Resistive cooling and laser cooling}

Due to the nature of the beryllium ion source and the applied injection technique, most often $\mathrm{Be}^{+}$and $\mathrm{Be}^{2+}$ were caught in the trap. Figure 4 shows a typical cooling curve for $\mathrm{Be}^{2+}$ (the number of $\mathrm{Be}^{2+}$ ions is constant on that time scale; therefore the plotted signal is proportional to the $\mathrm{Be}^{2+}$ temperature). Two different regions of the data were fitted with an exponential decay: the first region is $0 \mathrm{~s}-2200 \mathrm{~s}$ and the second is $2300 \mathrm{~s}-2800 \mathrm{~s}$. The two different exponential decays fit the data well. Cooling the axial motion of the $\mathrm{Be}^{2+}$ reduces the temperature of the two radial degrees of freedom as well as all degrees of freedom of the $\mathrm{Be}^{+}$. Note that the cooling laser was on at all times for this experiment but at high temperatures it has a negligible effect on the cooling. At about $2300 \mathrm{~s}$ a drastic change in the cooling rate occurs: laser cooling becomes effective and the drop in temperature becomes faster. In this phase the cooling is being done by the two radial degrees of freedom of the $\mathrm{Be}^{+}$but still the axial

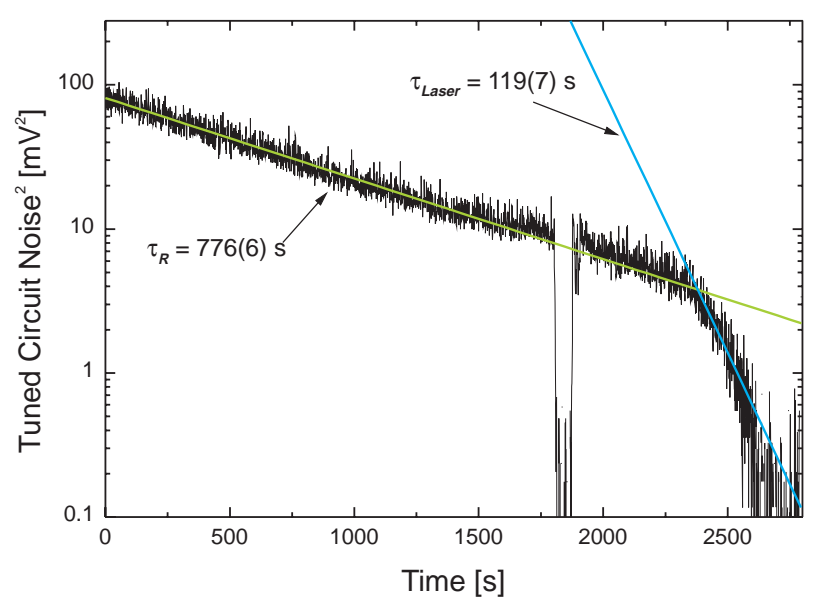

FIGURE 4. Tuned circuit noise signal of $\mathrm{Be}^{2+}$ ions stored with $\mathrm{Be}^{+}$ions. At first $\mathrm{Be}^{2+}$ is cooled mainly resistively with the tuned circuit. $\mathrm{Be}^{+}$is cooled mostly by the $\mathrm{Be}^{2+}$ via Coulomb collisions. As soon as the temperature is low enough for the laser cooling to be effective (at about $2300 \mathrm{~s}$ ), laser cooling of $\mathrm{Be}^{+}$becomes faster and the $\mathrm{Be}^{2+}$ is now cooled mainly by the $\mathrm{Be}^{+}$, indicated by the kink in the noise signal. 
signal of the $\mathrm{Be}^{2+}$ is observed. This is a first indication of sympathetic cooling of the monitored $\mathrm{Be}^{2+}$ by the laser cooled $\mathrm{Be}^{+}$.

The dip in the data at about $1800 \mathrm{~s}$ is due to tuning the ions off resonance to find the background level for background subtraction. The background is due to the Johnson noise across the input resistor of the amplifier.

\section{Sympathetic cooling of $\mathrm{Xe}^{q+}$}

Sympathetic cooling has already been seen in Fig. 4 involving $\mathrm{Be}^{+}$and $\mathrm{Be}^{2+}$. Sympathetic cooling of $\mathrm{Xe}^{q+}$ $(q>30)$ by $\mathrm{Be}^{2+}$ is shown in Fig. $5 . \mathrm{Be}^{2+}$ ions were loaded into the trap and soon thereafter $\mathrm{Xe}^{44+}$ ions were captured in the same trap. After $10 \mathrm{~s}$ the potential of the ring electrode was swept through the resonance of the $\mathrm{Be}^{2+}$ and the HCIs to probe the charge distribution. The maximum axial energy $E_{\max }$ that an ion of charge $q$ can have in a potential well of depth $U$ is given by:

$$
E_{\max }=q U=\frac{1}{2} k_{B} T \text {. }
$$

Since $U$ is the same for both $\mathrm{Be}^{2+}$ and $\mathrm{Xe}^{44+}$, the $\mathrm{Be}^{2+}$ temperature $T$ can be a factor of 20 lower than the $\mathrm{Xe}^{44+}$ temperature. Here $k_{B}$ is the Boltzmann constant. Both species will equilibrate collisionally. Ions with large axial amplitude (i.e. hot ions) have a lower oscillation frequency and need a deeper potential well to be on resonance with the tuned circuit. This causes the detected signal to be broadened towards higher voltages $V_{0}$. If

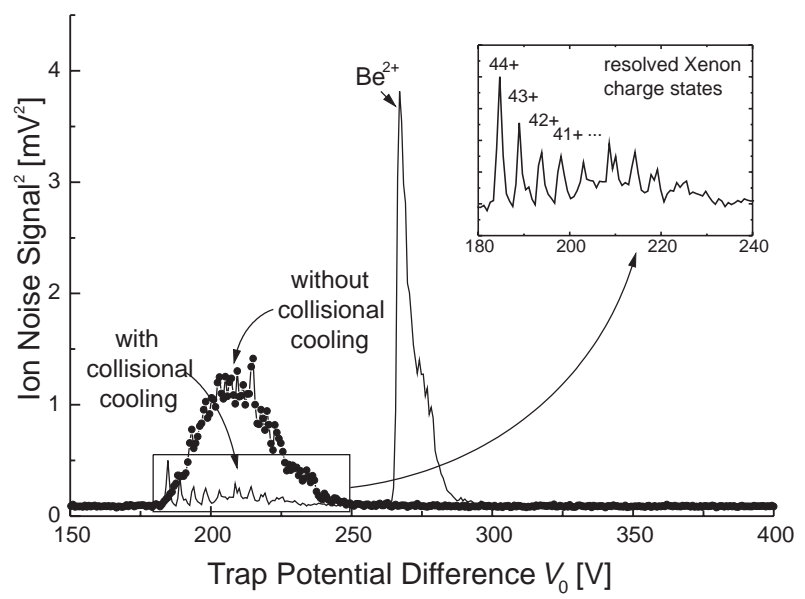

FIGURE 5. The tuned circuit signal indicates sympathetic cooling of $\mathrm{Xe}^{q+}$ ions. Without the presence of $\mathrm{Be}^{2+}$ a broad peak due to a wide energy distribution is visible when hot HCIs are captured (plot with symbols). When HCIs are merged into $\mathrm{Be}^{2+}$, a set of distinct peaks appear, revealing the charge state distribution of the colder xenon ions. the $\mathrm{Be}^{2+}$ ions are in the trap as well then the distribution starts to show a structure which corresponds to the individual charge states of the HCIs and the signal of the $\mathrm{Be}^{2+}$ ions can be seen as well. This is a strong indication of sympathetic cooling of HCIs by $\mathrm{Be}^{2+}$.

The next step is to catch the HCIs into a laser cooled beryllium plasma $(T<2 \mathrm{~K})$. Two different methods of detecting the ion cloud have been chosen and applied and a third method is being developed. A photomultiplier tube (PMT) detects scattered light through the ring electrode perpendicular to the cooling beam and is opposite a cryogenically cooled CCD camera that takes side view images of the fluorescing cloud. The PMT provides the time resolution whereas the CCD is an integrating detector that gives spatial information about the cloud.

Once the beryllium ions are cold, HCIs can be caught in the same fashion as explained above. This involves ramping and pulsing of certain electrodes. This can be a heat source and if so, an increase in the PMT signal should be observed. In Fig. 6 a PMT signal is displayed while two HCI loading sequences were done; the first sequence with no HCI beam coming from EBIT (short duration of higher scatter rate), then a second with loading of EBIT ions into the cold beryllium plasma. For the latter case the $\mathrm{Be}^{+}$ions heat up more than in the first case, since the additional energy of the $\mathrm{HCI}$ is transferred to the $\mathrm{Be}^{+}$.

The CCD camera gives additional information for the capture of HCIs into the cold beryllium plasma. In

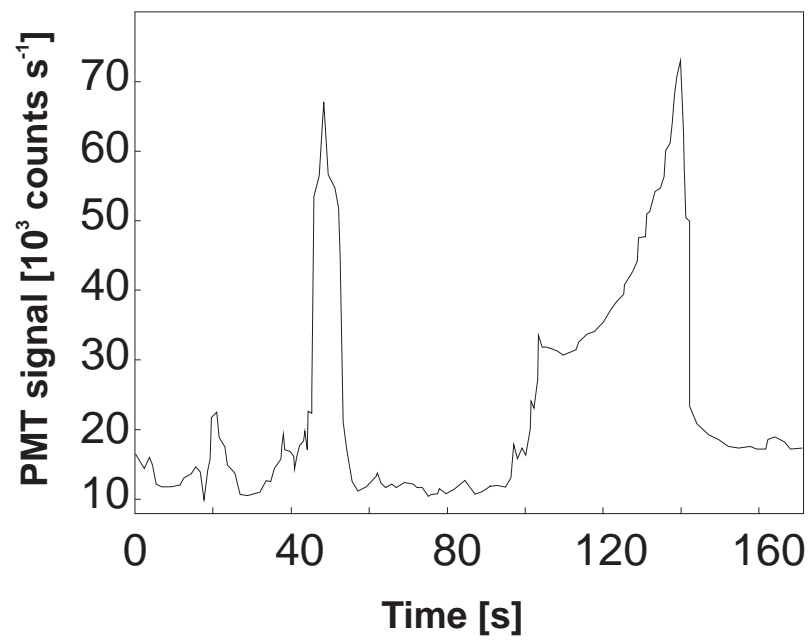

FIGURE 6. Signal of the photomultiplier tube. Laser cooled $\mathrm{Be}^{+}$was in the trap. At the first peak (50 s) the electrodes were pulsed for xenon capture, but no xenon was admitted. In the second peak (100s-140s) xenon was captured into the trap. It can be seen that the $\mathrm{Be}^{+}$ions were heated more (PMT shows a high scatter rate for a longer time) in the case when xenon ions were injected into the pre-cooled ions in the trap. 
Fig. 7 a) a side view of a cold $\mathrm{Be}^{+}$cloud can be seen. The two spots can be explained in the following way: As mentioned before $\mathrm{Be}^{+}$and $\mathrm{Be}^{2+}$ ions are typically caught in the same trap but only $\mathrm{Be}^{+}$is visible since there are no transitions excited for $\mathrm{Be}^{2+}$. As the ions cool they start to centrifugally separate due to different mass to charge ratios (13). $\mathrm{Be}^{+}$forms an annulus around the $\mathrm{Be}^{2+}$. Since the laser beam has a diameter close to the extent of the $\mathrm{Be}^{2+}$ cloud (i.e. the gap between the spots in Fig. 7 a) only two small sections of the $\mathrm{Be}^{+}$annulus can be observed (Fig. 8).

If HCIs $(m / q \approx 3)$ are introduced into the trap, they are expected to concentrate in the center. Therefore they would displace some of the $\mathrm{Be}^{2+}$ and make the gap between the two spots even bigger. This is what can be seen in Fig. 7 b).

A third method of visualizing the HCIs in the trap would be to project them onto a CCD chip located in the fringe field of the magnet below the trap. The detection efficiency of a HCI impacted on a CCD is very high compared to a low charge state ion (e.g. $\mathrm{Be}^{+}$or $\mathrm{Be}^{2+}$ ). This opens the possibility to check for HCIs in the trap, since the CCD would be almost 'blind' for beryllium ions
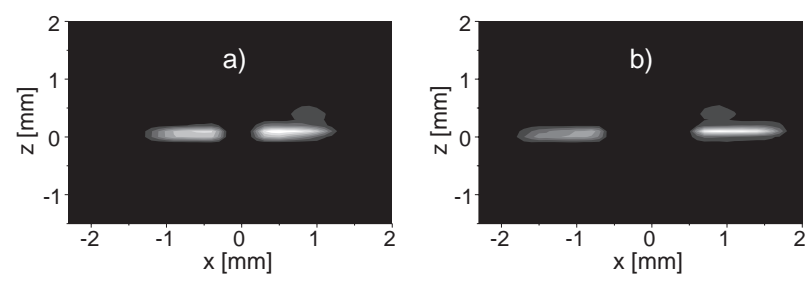

FIGURE 7. $\mathrm{CCD}$ side view pictures of the cold $\mathrm{Be}^{+}$cloud. In a) there were only $\mathrm{Be}^{+}$and $\mathrm{Be}^{2+}$ in the trap. In b) highly charged xenon ions were added. It can be seen that the gap between the two light spots increases. This indicates a radial displacement of the $\mathrm{Be}^{2+}$ ions by the additional xenon ions.

\section{TOP VIEW}

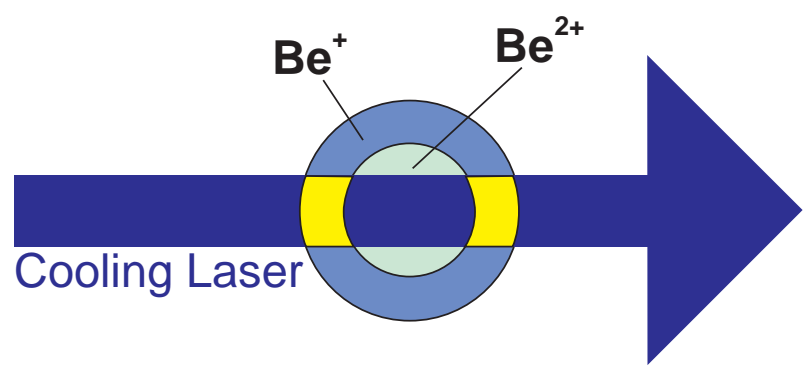

FIGURE 8. Schematic top view of the cold $\mathrm{Be}^{+}-\mathrm{Be}^{2+}$ cloud. Only the $\mathrm{Be}^{+}$cloud overlapping with the laser beam can scatter light (indicated by the lighter areas in the $\mathrm{Be}^{+}$cloud) producing two spots in the side view images. but would see every single HCI. The development of this method is in progress.

In HCIs, fine and hyperfine structure transitions of certain ions can be accessible with a laser (10), which would make an additional detection method feasible. The HCI cloud might be imaged directly with a CCD camera.

\section{Coulomb coupling parameter $\Gamma$}

To characterize the plasma the density and temperature has to be determined. For cases where only $\mathrm{Be}^{+}$was caught in the trap, the aspect ratio of the ellipsoidal cloud can be measured from the side view images from which the plasma density can be determined (11). Since the volume can be calculated from the same image, the number of trapped cold $\mathrm{Be}^{+}$ions can be determined (typical: $\left.4 \cdot 10^{5}\right)$. With this the number of $\mathrm{Be}^{2+}$ and $\mathrm{Xe}^{q+}$ ions can be extracted from the tuned circuit signal, which gives the relative numbers of ions assuming that the ions are in thermal equilibrium. The number of xenon ions was determined to be $\approx 400$. This is in good agreement with the number of counted ions after release from the trap when there was no beryllium in the trap.

Assuming the fluid model is applicable for this plasma a minimum density for the ions in the trap (11) can be obtained $\left(3 \cdot 10^{7} \mathrm{~cm}^{-3}\right.$ for $\left.\mathrm{Xe}^{44+}\right)$.

The temperature has been estimated in different ways: The cooling laser has been swept over the resonance and the measured FWHM of the fluorescence signal was about $300 \mathrm{MHz}$. This indicates a temperature of $1.7 \mathrm{~K}$. Another indication of the temperature is the numerical simulation of a mixture of $\mathrm{Be}^{+}$and $\mathrm{Be}^{2+}$ ions in a Penning trap. The mixture starts separating at around $0.25 \mathrm{~K}$ (12). And a third possibility is to use a model developed by O'Neil (13), predicting a separation at temperatures below $0.5 \mathrm{~K}$ based on a characteristic scaling length.

If a temperature of $1.7 \mathrm{~K}$ and a minimal density of $3 \cdot 10^{7} \mathrm{~cm}^{-3}$ is assumed the Coulomb coupling parameter $\Gamma$ for $\mathrm{Xe}^{44+}$ can be calculated:

$$
\Gamma=\frac{q^{2}}{4 \pi \varepsilon_{0} a_{0}} \frac{1}{k_{B} T}
$$

with $a_{0}=\left(\frac{3}{4 \pi n}\right)^{1 / 3}$. For an infinitely large onecomponent plasma theory predicts a crystallization at $\Gamma=172$. This means that the plasma in RETRAP should have crystallized. Therefore the effects of an ordered structure can expected to be seen when the cloud of strongly coupled HCIs is projected onto the CCD chip below the trap. 


\section{CONCLUSION}

With the described setup it was possible to trap several hundred HCIs in RETRAP and cool them to temperatures below $2 \mathrm{~K}$. Assuming the fluid model is valid the minimum density of these ions is estimated to $3 \cdot 10^{7} \mathrm{~cm}^{-3}$. This gives a Coulomb coupling parameter exceeding 1000 , indicating a highly charged ion crystal in the trap.

\section{ACKNOWLEDGMENTS}

We would like to thank J. W. McDonald for the excellent preparation of the ion beam from EBIT and D. Nelson and E. Magee for the technical support.

This work was performed under the auspices of the U.S. Dept. of Energy by the Lawrence Livermore National Laboratories under contract \#W-7405-ENG-48 and supported by the Texas Advanced Research Program.

\section{REFERENCES}

1. Moore, R. B., Hyp. Int. 81, 45-70 (1993)

2. Van Horn, H. M., Astr. J. 151, 227, 1968;

Van Horn, H. M., Strongly Coupled Plasma Physics, Rochester, NY, University of Rochester Press, 1990, 3-19;

3. Diedrich, F., Peik, E., Chen, J. M., Quint, W., Walther, H., Phys. Rev. Lett. 59, 2931-2934 (1987);

Wineland, D. J., Bergquist, J. C., Itano, W. M., Bollinger, J. J., Manney, C. H., Phys. Rev. Lett. 59, 29352938 (1987);

4. Brush, S. G., Sahlin, H. L., Teller, E., J. Chem. Phys. 45, 2102-2118 (1966);

Slattery, W. L., Doolen, G. D., DeWitt, H. E., Phys. Rev. A 26, 2255-2258 (1982)

5. Marrs, R. E., Levine, M. A., Knapp, D. A., Henderson, J. R., Phys. Rev. Lett. 60, 1715-1718 (1988);

Levine, M. A., Marrs, R. E., Henderson, J. R., Knapp, D. A., Schneider, M. B., Phys. Scr. T22, 157-163 (1988)

6. Schneider, D., DeWitt, D. E., Clark, M. W., Schuch, R., Cocke, C. L., Schmieder, R., Reed, K. J., Chen, M. H., Marrs, R. E., Levine, E., Fortner, R., Phys. Rev. A 42, 38893895 (1990)

7. Schneider, D., Church, D. A., Weinberg, G. M., Steiger, J., Beck, B. R., McDonald, J. W, Magee E., Knapp, D., Rev.Sci.Instrum 65, 3472-3478 (1994)

8. Bergquist, J. C.,Itano, W. M., Wineland, D. J., Phys. Rev. A 36, 428-430 (1987)

9. Walls, F. L., Dehmelt, H. G., Phys. Rev. Lett. 21, 127-131 (1968)
10. Klaft, I., Borneis, S., Engel, T., Fricke, B.,Grieser, R., Huber, G., Kühl, T., Marx, D., Neumann, R., Schröder, S., Seelig, P., Völker, L. Phys. Rev. Lett. 73, 2425-2427 (1994); Creso López-Urrutia, J.R., Beiersdorfer, P., Savin, D., Widmann, K., Phys. Rev. Lett. 77, 826-829 (1996);

Seelig, P. et al. to be published;

11. Brewer, L. R., Prestage, J. D., Bollinger, J. J., Itano, W. M., Larson, D. J., Wineland, D. J., Phys. Rev. A 38, 859-873 (1988)

12. DeWitt, H. E., Pollock, R., priv. comm., (1998)

13. O’Neil, T. M., Phys. Fluids 24, 1447-1451 (1981) 